\title{
Facial Target Detection and Recognition Algorithm Based on Information Fusion
}

\author{
Huayong Yang ${ }^{\text {a }}$, Huali Zhang \\ City College, Wuhan University of Science and Technology, Wuhan 430083, China \\ aYang_welcome@163.com
}

Keywords: Information fusion; Facial detection; Principal analysis.

\begin{abstract}
This paper is based on the idea of information fusion, which is proposed to use multi-information fusion technology to summarize the facial target detection technology and take it as a breakthrough point, by means of analyzing the strategy of common information fusion, it discusses the algorithm of facial detection based on analysis and principal component analysis (PCA) for facial recognition.
\end{abstract}

\section{Introduction}

Facial detection and recognition technology has great prospect in the application of public security, security authentication, bank, customs, video conference, transportation and so on. At the same time, with the development of society, as well as the increase of the speed and the flowing region, plus the increasing flow of world population, the facial detection and recognition technology has played an important role in the management of the floating population and statistical areas. Moreover, facial detection and recognition technology has great application prospect in the digital entertainment; facial detection and recognition technology can be used for identity authentication, monitoring people in public places, making image database retrieval so as to improve the interaction ability between human and computer. In many important occasions, such as security systems, credit card validation, criminal identification, customs transition check, which needs to use reliable facial detection and recognition with no harm and limitation for human beings.

\section{The Overview of Facial Target Detection Technology}

The Technology of Face Detection. Face detection refers to the process of finding out all the location, size, and pose of face during the period of inputting image (if it is existed). Due to the changes of scene, light, gesture and facial expressions (front, side), as well as some other reasons, it makes face detection very challenging, the realization of face detection method in general use is not realistic. Thus, solving the problem of face detection is still a main topic under the specific constraint conditions with certain application background in the field. The problem of face detection is based on image source, color, and lens, number of face, gesture, the complex degree of background and the fields of applications, which can be classified in many different. The researching object in the detection system of this paper is a dynamic color image with a frontal face acquired by a general camera, whose background is not too complex and its change is not too violent.

The Technology of Face Recognition. Face recognition refers to the process to find out the location, size, pose of all faces during the period of inputting image (if it exists) . Face recognition is a very difficult problem in the field of pattern recognition and computer vision, which is mainly displayed in the following aspects: the change of the facial expression and gesture, there is a close relationship between the facial pose and camera, as well as the position of the image. As for the face, it will have the gesture of front, side, rotation as well as the gesture of next swinging up and down, etc., at the same time the facial features such as eyes and other possible can be fully or partially occluded; since the facial feature is uncertain, face may have the change of hair style;moreover, the change of expression is often occurred, because people may have different kinds of expressions, which will directly affect the face detection and recognition; besides, the environmental change of the 
image, such as lighting, or the sensitivity of camera, as well as the change of optical axis and so on. All the above factors will cause the image to be unstable, which will increase the difficulties of making face detection and recognition.

\section{A Common Strategy for Information Fusion}

Information fusion is also called data fusion, which refers to the process to get more accurate and more complete information so as to complete the required decision-making and evaluation from the automatic analysis and synthesis of different knowledge sources and sensors to collect data with a certain criterion from multi levels. In the technology of biometric identification, using one single biometric recognition system often exists the following problems: it is easy to be influenced by the noise in signal acquisition, its biological characteristics is without universality, its biological feature is not easy to be distinguished, the recognition accuracy is not high and vulnerable to be attacked and camouflaged. Therefore, the identifying biological information fusion from different sources is an effective method to solve the above problems.

\section{Analysis of Face Detection Algorithm}

Sung and Poggio assumed that the face area can be composed of a series of small number of ellipsoid to have approximate simulation. The training samples can be divided into clusters (such as the front light can light the portrait of the left half side of face, taking it as a cluster of front image, but the light from the left to the right can be regarded as a cluster, etc.) Thus, it can calculate out the centroid of each cluster (according to the distribution of the gray value as the distribution of the quality to calculate), as well as the distance between its centroid cluster members to the Mahalanoblis distance. If all the cluster members are ellipsoid and the training samples can represent the goals of the targeted face, then the distance from a new face image to a centroid cluster's Mahalanoblis distance should be less than the distance from the center of the cluster to the largest part of its distance. Similarly, the distance from the non- face region point to each cluster centroid distance should be more than the distance from the most of the face image point from to the centroid distance.

Based on the above analysis, the algorithm of the face detection can be described as follows:

Learning Algorithm. Assume that a set of $S=\{F i \mid 1 \leq i \leq n\}$ is the vector for facial feature.

(1) $\mathrm{S}$ is divided into $\mathrm{m}$ clusters $\{\mathrm{Cj} \mid 1 \leq \mathrm{i} \leq \mathrm{m}\}$;

(2) Calculating out $\mathrm{Mj}$ of the centroid of each cluster $\mathrm{Cj}$, calculating out the inverse covariance matrix sigma $\mathrm{J}$, calculating out the distance between $\mathrm{Cj}$ to $\mathrm{Mj}$, $\mathrm{Dj}$ is defined as the the smallest radius of ellipsoid with the center of $\mathrm{Mj}$, which has the corresponding $\mathrm{p} \%$ in the distribution of the cluster $\mathrm{Cj}$.

\section{Detection Algorithm.}

(1) Gets a set $\{\mathrm{Mj}, \Sigma \mathrm{j}, \mathrm{Dj} \mid 1 \leq \mathrm{i} \leq \mathrm{m}\}$;

(2) If the detected image has a rows and B columns, the algorithm is as follows:

For scale $=$ Min; Scale $\leq$ Max; Scale $=$ Scale + ScaleStep Begin

For $\mathrm{i}=1 ; \mathrm{i} \leq \mathrm{A} ; \mathrm{i}=\mathrm{i}+\mathrm{t}$ Step

Begin

For $\mathrm{j}=1 ; \mathrm{j} \leq \mathrm{B} ; \mathrm{j}=\mathrm{j}+\mathrm{tStep}$

Begin

If the feature window $\mathrm{W}$ is totally in the tested image

Begin

(1) Extracting the window width of $\mathrm{W}$ Width=Scale; height eight $=4 *$ Scale/3, the center point is in (i, j);

(2) Extracting the feature vector from window W;

(3) Calculating out of the minimum distance from the center of mass F to Mio;

(4) If the distance between $\mathrm{F}$ and Mio is less than the distance between Dio Then

Window $\mathrm{W}$ contains a face 
Else Window W contains more than a face

End;

End;

End;

End;

\section{Face Recognition Based on Principal Component Analysis (PCA)}

The principal component analysis method is to realize the diagonalization of the whole scatter matrix by means of solving the eigenvalue. Assuming that the face images are taken as the training samples by dealing with the value of gray histogram, then arranging each image according to the order of all the pixels in the images, then a set of column vectors can be acquired: $\left\{X_{1}, X_{2}, \ldots \ldots . X_{N}\right\}, N$ can represent the number of facial images, the scatter matrix of the samples of facial images as well as the whole scatter matrix $S_{t}$ value can be expressed as:

$$
\begin{aligned}
& \bar{X}=\frac{1}{N} \sum_{i=1}^{N} X_{i} \\
& S_{t}=\frac{1}{N} \sum_{i=1}^{N}\left(X_{i}-\bar{X}\right)\left(X_{i}-\bar{X}\right)^{T}
\end{aligned}
$$

To solve the eigenvalue:

$\lambda \mathrm{w}=\mathrm{Stw}$

Descending the order of the feature value after decomposition, selecting the first $m$ of the non zero eigenvalue as well as its corresponding eigenvector as the principal component, the original face image can be described by the projection coefficient of low dimension space as follows:

$$
y=\sum_{i=1}^{m} a_{i} w_{i}
$$

Among them, $(\alpha 1, \alpha 2, \ldots \ldots \alpha m)$ can represent the position of the training samples of face image in the feature subspace, with the method of principal component analysis, according to the corresponding feature value to select the size of the main element, trying to choose the larger eigenvalues as possible as it can, for the practical face recognition, studying how many eigenvalues is most appropriate, usually in accordance with the two standards to determine the number of the characteristic value, the ratio of the characteristic value that is selected and all the eigenvalues should be more than or less than 0.9 , when ratio of the corresponding characteristic value and the maximum eigenvalue is less than a set value, it will be abandoned.

\section{Experiment and Analysis}

In order to prove the efficiency of the algorithm, two images are tested independently and use Matlab R2008a to do the experiment. The first chosen picture is a Lena image $(490 \times 490)$ overlaid with Gaussian noise $(\mu=0, \rho=0.02)$. The second image is a PCA image $(210 \times 350)$. At first, we use the traditional OSTU segmentation and the MP-PCA to segment the image as Figure 2.Then, as Table 1 shows, process the two images with the iterative method, PCA algorithm and MP-PCA algorithm to get the optimal threshold vector and compare the time that they use.
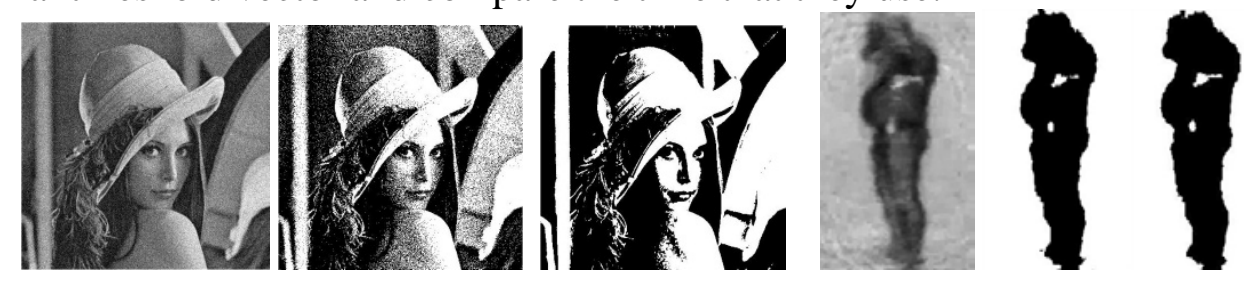

Fig.1 the processed images 
Table 1 performance of algorithms

\begin{tabular}{ccccc}
\hline & \multicolumn{2}{c}{ Lena } & & PMMW image \\
\hline Algorithm & Time(s) & Optimal Threshold Vector & Time(s) & Optimal Threshold Vector \\
\hline Iterative & 137.97 & $(185.127)$ & 87.45 & $(124.27)$ \\
PSO & 1.425 & $(185.127)$ & 0.735 & $(124.27)$ \\
MP-PSO & 0.644 & $(185.127)$ & 0.379 & $(124.27)$ \\
\hline
\end{tabular}

Results analysis. We can conclude from Fig.1 that MP-PCA can make the processed image more accurate and stable. Data in Table 1 obviously shows: compared with the iterative method, MP-PCA takes less time, because swarm of particles updates themselves by tracking individual and global extremum during each iteration. Compared with PCA, MP-PCA adopts AEWV model to guide particles searching optimal global solution, therefore the speed of that is doubled.

\section{Conclusion}

Due to the non-rigid feature of the facial object, influenced by the similarity and various changeable factors, realizing the accuracy of face recognition is still very difficult. With the development of image processing, pattern recognition, artificial intelligence and biological psychology research, the technology of facial detection and recognition will obtain greater development.

\section{Acknowledgments}

This paper was supported by the "Department of Education of Hubei Province: 2015 Annual Scientific Planning Projects B2015358”

\section{References}

[1] Lin S H, Kung S Y and Lin L J. 1997, Face recognition/detection by probabilistic decision-based neural network. IEEE Transactions on Neural networks, vol.8, pp114-132.

[2] Brunelli R, Poggio T. 1993, Feature recognition: Feature versus templates. IEEE Transactions on PAMI, vol.15, pp1024-1052.

[3] Huang C L, Chen C W. 1992, Human facial feature extraction for face interpretation and recognition. Pattern Recognition, vol.25, pp1435-1444.

[4] Lee S Y, Ham Y K and Park R H. 1996, Recognition of human front faces using knowledge-based feature extraction and neuro-fuzzy algorithm. Pattern Recognition, vol.299, pp1863-1876. 